\title{
An Optimization Technique for RF Buffers with Active Inductors
}

\author{
Tai-Cheng Lee \\ Graduate Institute of Electronics Engineering and \\ Department of Electrical Engineering \\ National Taiwan University \\ Taipei 10617, Taiwan \\ Email: tlee@cc.ee.ntu.edu.tw
}

\begin{abstract}
A technique of sizing RF buffers is presented to optimize with minimum power dissipation. By using the proposed current-reusing technique, the operating frequency of the RF buffer can be further increased with the same power consumption. The same technique can be also applied to reduce power by a factor of two while keeping the same operating frequency. The analysis is also verified by the circuit simulation.
\end{abstract}

\section{INTRODUCTION}

RF frequency synthesizers usually need to drive many circuits at the same time, such as up/down conversion mixers in both RF transmitters and receivers, shown in Fig. 1.

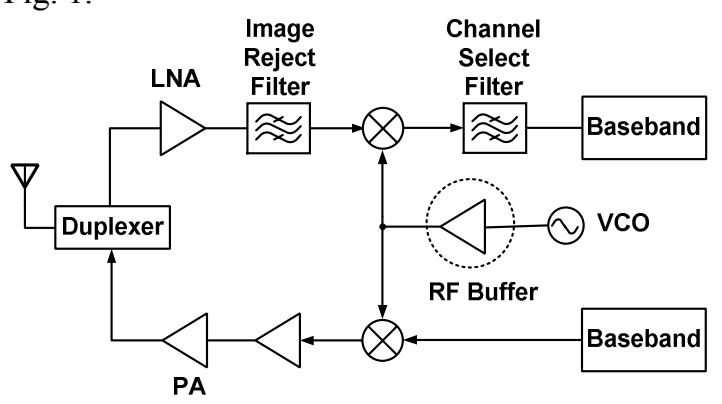

Fig. 1. A simplified RF transceiver architecture shows the necessity of inserting a RF buffer between the frequency synthesizer and mixers.

The voltage controlled oscillator (VCO) in the frequency synthesizer is sensitive to loading effect. If the VCO is an $L C$ resonator, large load capacitance reduces the resonant frequency. Thus, the tuning range becomes narrower and the carrier frequency shifts from the desired band. If the VCO is ring-oscillator type, large load capacitance may cause the ring oscillator fail to oscillate and the frequency synthesizer cannot work properly. Thus, inserting a buffer between the VCO and the up/down conversion mixers is necessary.

Adding a buffer can avoid the loading effect described above and can get an additional benefit from the isolation between the synthesizer and other noisy signals that may couple through the mixers. However, the buffer consumes large power to transfer the high-frequency signal to loads because the power consumption is proportional to $f C V^{2}$, where $f$ and $C$ are quite significant in $\mathrm{GHz}$ operation. Therefore, minimizing the power dissipation of the buffer is a major issue.

\author{
Yen-Chuan Huang \\ Graduate Institute of Electronics Engineering and \\ Department of Electrical Engineering \\ National Taiwan University \\ Taipei 10617, Taiwan \\ Email: r2943007@ee.ntu.edu.tw
}

An analog filter can be combined in a buffer design to achieve the frequency selectivity. Analog filters can be constructed in many ways using resistors, capacitors and inductors. A parallel $L C$ circuit is the bandpass filter prototype. However, passive inductors have the drawback of large size. Therefore, instead of using passive inductors, CMOS filters can incorporate active inductors to save the chip area.

The remains of this paper describe the active filter design strategy using active inductors. Section II is the analysis of the proposed active inductor structure including the method of minimizing the power consumption. Section III is the simulation results, and section IV summarizes with conclusions.

\section{ACTIVE FILTER DESIGN}

A gyrator with a capacitor $C$ can emulate an inductor in a parallel $L C$ filter [1]. Shown in Fig. 2, a gyrator is a $G_{\mathrm{m}}$ cell with a negative $G_{\mathrm{m}}$ cell on the feedback path.

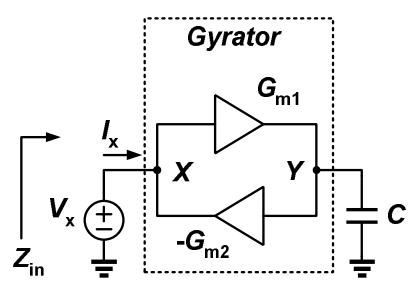

Fig. 2. A gyrator is composed by two transconductance cells.

The effective impedance $Z_{\text {in }}$ in Fig. 1 can be derived as Eq. (1)

$$
\begin{gathered}
-I_{X}=-G_{m 2}\left(G_{m 1} V_{X} \times \frac{1}{s C}\right) \\
\frac{V_{X}}{I_{X}}=\frac{s C}{G_{m 1} G_{m 2}}=s L
\end{gathered}
$$

where

$$
L=\frac{C}{G_{m 1} G_{m 2}}
$$

Equation (1) shows that the effective inductance of a gyrator is inverse proportional to the product of transconductance of the two $G_{\mathrm{m}}$ cells and proportional to the capacitor capacitance $C$. In RF buffer application, no extra capacitor but only the parasitic capacitance of $G_{\mathrm{m}}$ is at node Y. Therefore, to drive a large capacitive load in an RF buffer application, the inductance should be small enough to tune the center frequency to the desired value, i.e. the transconductance of the $G_{\mathrm{m}}$ cell should be large. 
In CMOS technology, the tranconductance can be increased either by using large aspect ratio devices or increasing the bias current but at the cost of more power consumption. Thus, for a limited power consumption constraint, the transconductance can be increased only by using large devices, but large devices induce more parasitic capacitance and lower the resonant frequency however. This contradiction complicates the active inductor design.

The quality factor $(Q)$ of an active inductor is directly proportional to output impedance of the $G_{\mathrm{m}}$ cell [1]. Very high- $Q$ active inductors achieved by cascode devices [2] or $Q$-enhancement topology [3], [4] are not suitable for RF buffer application because the buffer operates in large signals and large- $Q$ active inductors may cause instability. Also, noise power is $Q$ times worse in a resonant circuit employing an emulated inductor [1]. Thus very high- $Q$ active inductor design is not used. But, if the positive $G_{\mathrm{m}}$ cell in the gyrator is replaced by a simple common-gate amplifier, as shown in Fig. 3, the input admittance $Y_{\text {in }}$ is

$$
Y_{\text {in }} \approx s C_{p}+\frac{1}{R_{s}+s L}+\frac{1}{R_{p}},
$$

where

$$
\begin{gathered}
C_{p}=C_{d b 1}+C_{s b 2}+C_{g s 2}+\frac{C_{Y} C_{g d 1}}{C_{Y}+C_{g d 1}}, \\
R_{s}=\frac{1}{g_{m 1} g_{m 2}\left(r_{o 2} / / R_{o c}\right)}, \\
L=\frac{C_{g d 1}+C_{Y}}{g_{m 1} g_{m 2}}, \\
R_{p}=r_{o 1} / /\left(\frac{C_{g d 1}+C_{Y}}{g_{m 1} C_{g d 1}+g_{m 2} C_{Y}}\right) \approx \frac{1}{g_{m 2}} .
\end{gathered}
$$

Thus, the quality factor is poor due to the small equivalent impedance $R_{\mathrm{p}}$ in parallel with the active inductor and the filter provides poor frequency selectivity. Thus, an active inductor with reasonable $Q$ is suitable for RF buffer application.

\section{A. Proposed Active Inductor Design and Analysis}

Figure 4 shows the proposed the active inductor design. The differential-to-single-ended amplifier represents the positive $G_{\mathrm{m}}$ cell and the PMOS common-source amplifier forms the negative $G_{\mathrm{m}}$ cell in the gyrator structure. Both $G_{\mathrm{m}}$ cells have large output impedance. Therefore, without using other $Q$-enhancement circuit, the overall $Q$ factor is still larger than simply employing a common-gate amplifier.

If $M_{2,3}$ and $M_{4,5}$ are perfect symmetric, from Eq. (1), we obtain

$$
L_{\text {ind }}=\frac{C_{p}}{g_{m 1} g_{m 2}},
$$

where $C_{\mathrm{p}}$ denotes the total parasitic capacitance at the drain of transistor $M_{3}$.

In order to make the resonant frequency higher, we can increase $g_{\mathrm{m} 1}$ and $g_{\mathrm{m} 2}$ by increasing the sizing of the transistors $M_{1} \sim M_{3}$, but $C_{\mathrm{p}}$ is increased simultaneously. Thus, increasing the transconductance of the two amplifiers by increasing the width of the transistors arbitrarily can not ensure higher resonant frequency

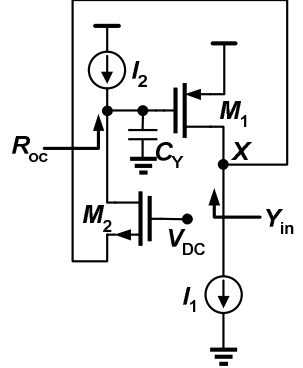

Fig. 3. A gyrator is composed by common-source and common-gate amplifiers.

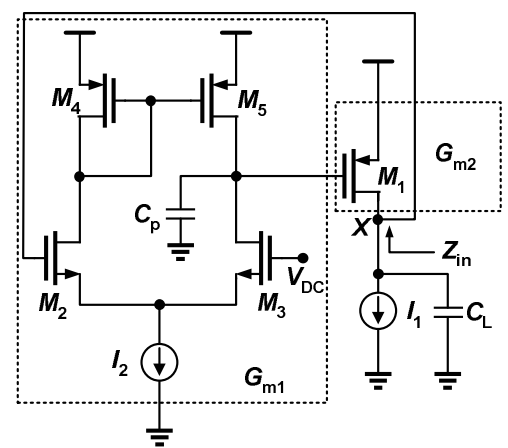

Fig. 4. Proposed active inductor design (only half circuit is shown).

eventually.

The parasitic capacitance $C_{\mathrm{p}}$ consists of the parasitic capacitance of the transistors $M_{1}, M_{3}$ and $M_{5}$, and can be written as follows.

$$
C_{p}=C_{g s 1}+C_{d b 3}+C_{d b 5}+C_{g d 1}+C_{g d 3}+C_{g d 5}
$$

Assuming all the transistors operate in the saturation region, the gate-to-drain overlap capacitors are much smaller than the other parasitic capacitors and can be neglected. Thus, expression (3) becomes

$$
\begin{aligned}
C_{p} & \approx C_{g s 1}+C_{d b 3}+C_{d b 5} \\
& \approx \frac{2}{3} W_{1} L_{1} C_{o x}+\gamma_{3} W_{3}+\gamma_{5} W_{5} \\
& =\frac{2}{3} W_{1} L_{1} C_{o x}+\gamma_{2} W_{2}+\gamma_{5} W_{5} .
\end{aligned}
$$

Equation (4) also uses average capacitance for $C_{\mathrm{db} 3}$ and $C_{\mathrm{db} 5}$. Thus, the junction capacitance is simply proportional to the width of the transistor with a constant coefficient $\gamma$, determined by the process parameters.

From Eqs. (2) and (4), the effective inductance becomes

$$
\begin{aligned}
L_{i n d}=\frac{C_{p}}{g_{m 1} g_{m 2}} & =\frac{\frac{2}{3} W_{1} L_{1} C_{o x}+\gamma_{2} W_{2}+\gamma_{5} W_{5}}{\sqrt{2 \mu_{p} C_{o x} \frac{W_{1}}{L_{1}} I_{1}} \sqrt{2 \mu_{n} C_{o x} \frac{W_{2}}{L_{2}} \frac{I_{2}}{2}}} \\
= & \frac{\frac{2}{3} W_{1} L_{1} C_{o x}+\gamma_{2} W_{2}+\gamma_{5} W_{5}}{\sqrt{2 \mu_{p} \mu_{n}} C_{o x} \times \sqrt{\frac{W_{1}}{L_{1}}} \sqrt{\frac{W_{2}}{L_{2}}} \sqrt{I_{1} I_{2}}} .
\end{aligned}
$$

The total capacitance $C_{\mathrm{x}}$ at node $X$ can be estimated as

$$
\begin{aligned}
C_{x} & \approx C_{L}+C_{g s 2}+C_{d b 1} \\
& \approx C_{L}+\frac{2}{3} W_{2} L_{2} C_{o x}+\gamma_{1} W_{1},
\end{aligned}
$$

where $C_{\mathrm{L}}$ represents the total load capacitance including 
the current source parasitic capacitance. Equation (6) neglects gate-to-drain overlap capacitance also.

If all the transistors have minimum channel length, $L_{\text {min }}$, multiply (5) by (6) we get

$$
L_{i n d} C_{x}=\frac{\left(\frac{2}{3} L_{\min } C_{o x} W_{1}+\gamma_{2} W_{2}+\gamma_{5} W_{5}\right)\left(\gamma_{1} W_{1}+\frac{2}{3} L_{\min } C_{o x} W_{2}+C_{L}\right)}{\left(\frac{C_{o x}}{L_{\min }} \sqrt{2 \mu_{p} \mu_{n}} \sqrt{I_{1} I_{2}}\right) \sqrt{W_{1} W_{2}}}
$$

The resonant frequency of a parallel $L C$ filter is equal to the reciprocal of the square root of Eq. (7). Because transistor $M_{5}$ takes no effect on the transconductance of either $G_{\mathrm{m}}$ cell, minimum width of $M_{5}$ should be chosen to minimize the contribution of $C_{\mathrm{p}}$ as long as it can operate in the saturation region. Thus, if power consumption is limited (i.e. $I_{1}$ and $I_{2}$ both are fixed), only two parameters $W_{1}$ and $W_{2}$, the width of the transistors $M_{1}$ and $M_{2}$, are variable. Take partial derivatives on Eq. (7) with respect to $W_{1}$ and $W_{2}$, respectively, and set both expressions equal to zero.

$$
\begin{gathered}
\frac{\partial}{\partial W_{1}}\left(L_{i n d} C_{x}\right)=0 \\
\frac{\partial}{\partial W_{2}}\left(L_{i n d} C_{x}\right)=0
\end{gathered}
$$

Divide one resulting equation by another and through algebraic simplification, we get

$$
\begin{aligned}
& W_{1}\left(\frac{4}{3} L_{\min } C_{o x} \gamma_{1} W_{1}+\frac{2}{3} L_{\min } C_{o x} C_{L}+\gamma_{1} \gamma_{5} W_{5}\right) \\
& \quad=W_{2}\left(\frac{4}{3} L_{\min } C_{o x} \gamma_{2} W_{2}+\gamma_{2} C_{L}+\frac{2}{3} L_{\min } C_{o x} \gamma_{5} W_{5}\right) .
\end{aligned}
$$

Equation (10) indicates that the parameters $W_{1}$ and $W_{2}$ must meet this constraint equation to minimize $L_{\text {ind }} C_{\mathrm{x}}$ in Eq. (7). Typically, if $W_{1}, W_{2}$ and $W_{5}$ are not too large, the term containing load capacitance $C_{\mathrm{L}}$ dominates in the parenthesis of Eq. (10), therefore Eq. (10) can be simplified to

$$
\begin{gathered}
\frac{2}{3} L_{\min } C_{o x} C_{L} W_{1} \approx \gamma_{2} C_{L} W_{2}, \\
\frac{W_{1}}{W_{2}}=\frac{\gamma_{2}}{\frac{2}{3} L_{\min } C_{o x}} .
\end{gathered}
$$

Equation (11) shows that the width ratio between transistors $M_{1}$ and $M_{2}$ has a proportional relation with a constant proportional coefficient. This constant can be calculated from the process parameters and approximately equals to unity. Using the above approximation and go back to solve the Eq. (8), we obtain the following solution.

$$
W_{1} \approx \sqrt{\frac{3}{8} \frac{W_{5} C_{L} \gamma_{5}}{L_{\min } C_{o x} \gamma_{2}}}
$$

Combine with the Eqs. (11) and (12), the approximation of the optimal sizes that maximizing the resonant frequency can be found.

From another point of view, Eq. (7) can be modified to

$$
\sqrt{I_{1} I_{2}}=\frac{\left(\frac{2}{3} L_{\min } C_{o x} W_{1}+\gamma_{2} W_{2}+\gamma_{5} W_{5}\right)\left(\gamma_{1} W_{1}+\frac{2}{3} L_{\min } C_{o x} W_{2}+C_{L}\right)}{\left(\frac{C_{o x}}{L_{\min }} \sqrt{2 \mu_{p} \mu_{n}} L_{i n d} C_{x}\right) \sqrt{W_{1} W_{2}}} \cdot
$$

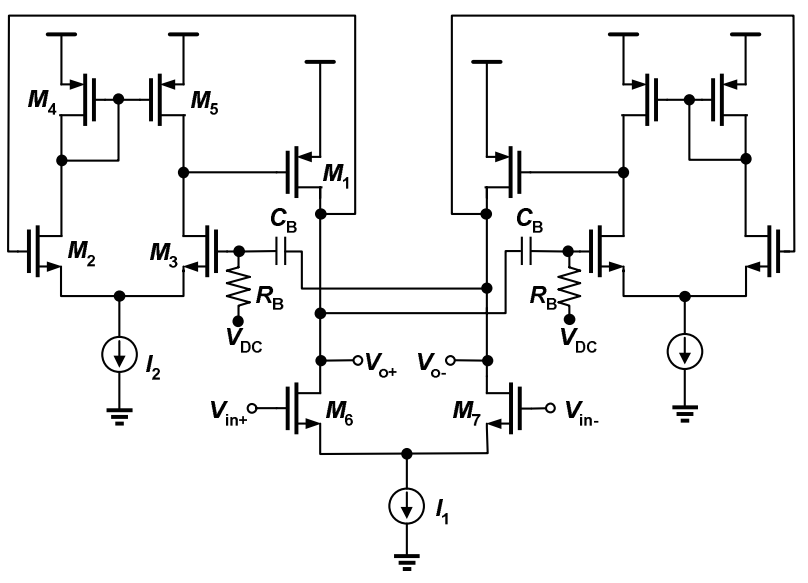

Fig. 5. An RF buffer uses active inductors as loads and the active inductors employ current reusing technique.

\begin{tabular}{|cc|}
\hline$L_{\min }$ & $0.35 \mu \mathrm{m}$ \\
$C_{\mathrm{ox}}$ & $4.6 \mathrm{e}-3 \mathrm{~F} / \mathrm{m}^{2}$ \\
$\gamma_{2}$ & $1.2363 \mathrm{nF} / \mathrm{m}$ \\
$\gamma_{5}$ & $1.7442 \mathrm{nF} / \mathrm{m}$ \\
$W_{5}$ & $20 \mu \mathrm{m}$ \\
$C_{\mathrm{L}}$ & $0.3 \mathrm{pF}$ \\
\hline
\end{tabular}

Table 1. Parameters used in MATLAB simulation.

For a desired resonant frequency (i.e. $L C$ product value is a given constant) similar to the previous analysis, the optimal width of $W_{1}$ and $W_{2}$ can be found to minimize (13). Throughout the calculation, the solution still follows (11) and (12). This indicates that while sizing the transistors based on Eqs. (11) and (12), minimum power can be used to produce the desired resonant frequency. This can be a minimum-power-consuming estimation for generating a specific resonant frequency.

\section{B. Current Reusing Technique}

If differential signal operation is available or necessary, the resonant frequency can be further increased without consuming additional power by changing the circuit in Fig. 4 as Fig. 5 shows.

In Fig. 5, both input ports of the differential-to-singleended amplifier connect to small signal inputs $V_{\mathrm{o}^{+}}$and $V_{\mathrm{o}-}$ which are different from that in Fig. 4, where one input port shorts to AC ground. $R_{\mathrm{B}}$ and $V_{\mathrm{DC}}$ set the operating points of the $G_{\mathrm{m}}$ cells and $C_{\mathrm{B}}$ is used to form AC coupling.

The active inductor property remains the same as before, but the effective inductance seen at node $V_{\mathrm{o}^{+}}$is reduced because the transconductance of the positive $G_{\mathrm{m}}$ cell increases by a factor of two due to the out-of-phase signal input now. Thus, change $g_{\mathrm{m} 2}$ in the Eq. (5) into $2 g_{\mathrm{m} 2}$, and apply the analysis as section A does, the same results (11) and (12) can be obtained. This means the sizing rules remain the same, but for a prescribed resonant frequency requirement, the power dissipation can be reduced by a factor of two in comparison to the conventional structure by using this technique.

\section{SIMULATION RESULTS}




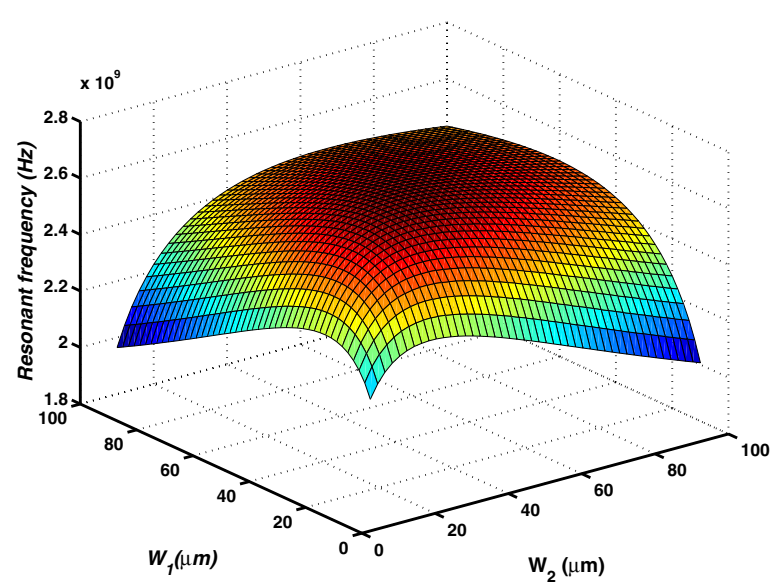

Fig. 6. Numerical plot of the resonant frequency with different transistor sizes.

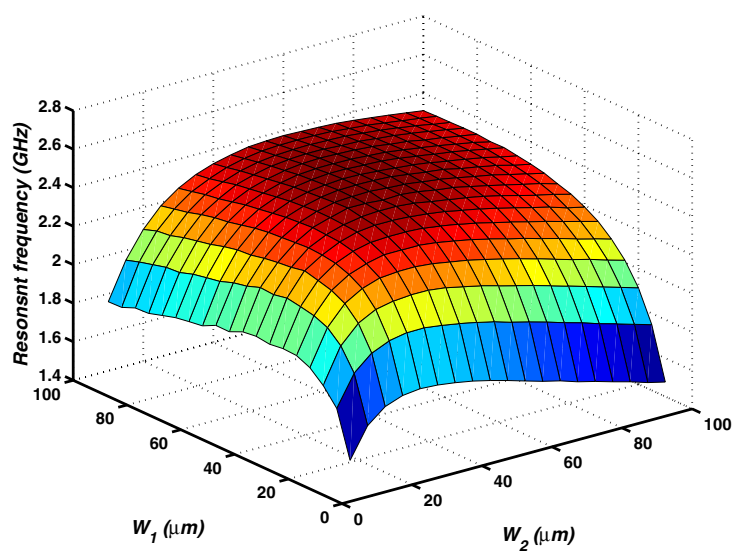

Fig. 7. Resonant frequency from SPICE simulation.

The solutions to the Eq. (7) are the optimal sizes for minimizing the power dissipation. MATLAB is used to predict the actual solutions. Fig. 6 is the plot of resonant frequency based on Eq. (7) with different $W_{1}$ and $W_{2}$ values, where the process parameters are calculated from the TSMC 0.35- $\mu \mathrm{m}$ CMOS process and summarized in Table 1. From Fig. 6, we find the optimal sizes in this case which are $39.6 \mu \mathrm{m}$ and $40.7 \mu \mathrm{m}$ for $W_{1}$ and $W_{2}$, respectively. The values of $W_{1}$ and $W_{2}$ calculated from Eqs. (11) and (12) are $44.4 \mu \mathrm{m}$ and $38.6 \mu \mathrm{m}$ which are a little different from the actual solutions. But, Fig. 6 shows that the resonant frequency changes little at the points adjacent to the optimal solution. Thus, Eqs. (11) and (12) can predict the solutions of Eq. (7) very well.

Use SPICE to simulate the circuit shown in Fig. 5 but without employing current-reusing technique. Fig. 7 shows the frequency where the buffer has maximum gain with different transistor width. Compared with Fig. 6, in Fig. 7 the resonant frequency varies more slowly with large $W_{1}$ and $W_{2}$ values. This is because we estimate the gate-to-source capacitance as $2 W L C_{\text {ox }} / 3$. However, this estimation becomes invalid for low $V_{\mathrm{GS}}$ as shown in Fig. 8. If the current flowing through the transistor $M_{1}$ is fixed, the overdrive voltage of $M_{1}$ will decrease by increasing $W_{1}$. Thus, for large $W_{1}$ values, $C_{\mathrm{gs} 1}$ makes less

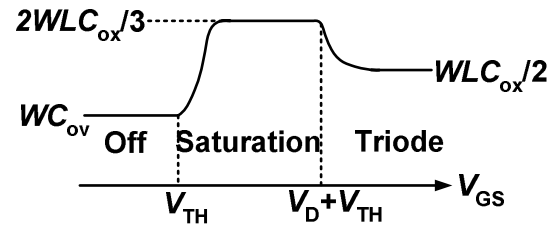

Fig. 8. Variation of gate-source capacitance versus $V_{\mathrm{GS}}$.

contribution to parasitic capacitance as Eq. (4) predicts. As a result, the resonant frequency is less sensitive to the transistor width variation. Although Eq. (7) can not precisely predict the resonant-frequency-variation characteristic with large $W_{1}$ and $W_{2}$ values, Eqs. (11) and (12) still give good approximation to the optimal sizes.

\section{CONCLUSIONS}

An RF buffer is used between the frequency synthesizer and its loads. The buffer consumes large power because high operating frequency and large load capacitance. The proposed buffer uses active inductors as a filter in order to provide frequency selectivity. A method for minimizing the power consumption of this buffer design is presented and verified by the circuit simulation. Finally, a current-reusing technique can be applied to save the power up to a factor of two.

\section{ACKNOWLEDGEMENT}

The authors would like to thank the support of National Science Council under grant NSC 93-2220-E-002 -010 and National Chip Implementation Center (CIC) for chip fabrication.

\section{REFERENCES}

[1] Yun-Ti Wang and Asad A. Abidi, "CMOS Active Filter Design at Very High Frequencies," in IEEE J. Solid-State Circuits, Vol. 25, No. 6, December 1990, pp.1562-1574.

[2] A. Thanachayanont and A. Payne, "VHF CMOS Integrated Active Inductor," Electronics Letters, vol. 32, No. 11, May 1996, pp. 999-1000.

[3] U. Yodprasit and J. Ngarmnil, "Q-Enhancing Technique for RF CMOS Active Inductor," in IEEE International Symposium on Circuits and Systems, May 2000.

[4] Markus Grözing, Andreas Pasht and Manfred Berroth, "A 2.5 V CMOS Differential Active Inductor with Tunable L and $\mathrm{Q}$ for Frequencies UP to $5 \mathrm{GHz}$," IEEE Radio Frequency Integrated Circuit (RFIC) Symposium, May 2001. 